\title{
Multi-objective performance optimization of ORC cycle based on improved ant colony algorithm
}

https://doi.org/10.1515/phys-2019-0006

Received Oct 30, 2018; accepted Jan 28, 2019

\begin{abstract}
To solve the problem of multi-objective performance optimization based on ant colony algorithm, a multi-objective performance optimization method of ORC cycle based on an improved ant colony algorithm is proposed. Through the analysis of the ORC cycle system, the thermodynamic model of the ORC system is constructed. Based on the first law of thermodynamics and the second law of thermodynamics, the ORC system evaluation model is established in a MATLAB environment. The sensitivity analysis of the system is carried out by using the system performance evaluation index, and the optimal working parameter combination is obtained. The ant colony algorithm is used to optimize the performance of the ORC system and obtain the optimal solution. Experimental results show that the proposed multi-objective performance optimization method based on the ant colony algorithm for the ORC cycle needs a shorter optimization time and has a higher optimization efficiency.
\end{abstract}

Keywords: Ant colony algorithm, ORC cycle, multiobjective performance optimization

PACS: $07.05 . R m, 84.70 .+p, 89.20 . F f$

\section{Introduction}

An Organic Rankine Cycle (ORC) uses organic matter with a low boiling point and high evaporation pressure as the

\footnotetext{
*Corresponding Author: Xinli Wei: School of Chemical Engineering and Energy, Zhengzhou University, Zhengzhou, 450001, China; Research Centre on the Technology and Equipments for Energy Saving in Thermal Energy System, Ministry of Education, Zhengzhou, 450001, China; Email: xlwei@zzu.edu.cn

Rong He: School of Chemical Engineering and Energy, Zhengzhou University, Zhengzhou, 450001, China; Email: herong.0220@foxmail.com

Nasruddin Hassan: School of Mathematical Sciences, Faculty of Science and Technology, Universiti Kebangsaan Malaysia UKM Bangi, Selangor, Malaysia; Email: nas@ukm.edu.my
}

Ә Open Access. (c) 2019 R. He et al., published by De Gruyter. (cc) BY License working fluid to recover and utilize various low-grade energy sources such as industrial waste heat, solar energy, geothermal energy, biomass energy, ocean temperature difference energy, and LNG cold energy. It has the advantages of simple structure, high efficiency, environmental friendliness and adaptability [1]. At present, the research on ORCs is mainly focused on the selection and optimization of the ORCs' working fluids. The performance evaluation index plays a very important role in ORC optimization. For ORCs with different working fluids, the choice of performance evaluation index is also different. The evaluation index is the core and key of the energy conversion system analysis method [2]. The ORC performance evaluation indicators in current use can be divided into two categories. One is the decrease of the single performance evaluation index based on the first law of thermodynamics, while the other is the single performance evaluation index based on the second law of thermodynamics [3]. However, the single objective optimization results are inconsistent with or even contradictory to the actual needs of the project. Therefore, ORC performance evaluation often involves multiple indexes [4]. At this point, the optimization of an ORC is to solve the multi-objective optimization problem. At present, there is not much research on multi-objective optimization of ORCs at home and abroad.

In the literature [5], a multi-objective optimization method for a traction power supply system based on improved particle swarm optimization algorithm is proposed [5]. A simulation method of vehicle network coupling system model and load flow distribution is used. A multi-objective optimization model for the traction power supply system is constructed with the main design principle as the constraint and the total capacity of the whole line and the minimum average power loss as the objectives. In order to improve the global search ability and convergence speed in the process of solving the model, a chaotic multi-objective particle swarm optimization algorithm based on Pareto entropy is designed. A fuzzy membership function is used to calculate the satisfaction degree of each objective function in the Pareto solution set to determine the final system optimization scheme. However, the optimization time is longer. In the literature [6], a 
cascade ORC system is proposed [6]. Thermodynamic models of two systems with and without diffluence are built. Taking evaporation temperature and output work as independent variable and objective function, respectively, a genetic algorithm is used to optimize the heat exchanger Comparison of system performance under two working conditions is carried out. The influence of the series on the performance of the system and the rules of cycle changes at all levels in the cascade system are analyzed. Split-flow technology can increase the optimum evaporation temperature of each heat exchanger, reduce the exergy loss of the preheater, and increase the performance of the system. However, the optimization efficiency of the method is low.

\section{Materials and methods}

\subsection{Establishment of component model for ORC cycle system}

The establishment of the thermodynamic model of an ORC system is the basis of its thermal analysis. Based on the first and second laws of thermodynamics, the model of each component of the system is established, and thus the performance evaluation model of the system. The ORC system consists of evaporator, expander, condenser and refrigerant circulating pump [7]. In the thermodynamic analysis of organic Rankine cycles, the condenser section of the system can be divided into three sections: precooling section, condensing section, and supercooling section. The evaporator part can also be divided into three sections: preheating section, evaporating section, and overheating section.

\subsubsection{Expander part}

The high-pressure organic working steam at the outlet of the evaporator enters the expander at the state point 1 . After the adiabatic expansion of the expander, the state point is 2. On the T-S diagram, the ideal exhaust point is 3, assuming that the work done by the organic working medium in the expander is an isentropic process. For the state point 3 ,

$$
S_{3}=S_{1}, P_{3}=P_{\text {cond }}
$$

where $P_{\text {cond }}$ is the condensing pressure of the system $(\mathrm{kPa})$ and $P_{2}=P_{\text {cond }}$.

Other state parameters of the state point 3 are given by

$$
T_{3}=\operatorname{refpropm}\left({ }^{\prime} T^{\prime},{ }^{\prime} P^{\prime}, P_{3},{ }^{\prime} S^{\prime}, S_{3}, F L U I D\right)
$$

$$
h_{3}=\operatorname{refpropm}\left({ }^{\prime} H^{\prime},{ }^{\prime} T^{\prime}, T_{3},{ }^{\prime} S^{\prime}, S_{3}, F L U I D\right)
$$

where FLUID is the organic working fluid of the system and $h_{3}$ is the specific enthalpy of the state point 3 . The refpropm function used in the calculation process is the REFPROP software function called by MATLAB [8], which is used to calculate the physical parameters of organic working substances at a certain state point.

For the state point 2, the specific enthalpy is given by

$$
h_{2}=h_{1}-\eta_{t}\left(h_{1}-h_{3}\right)
$$

where $\eta_{t}$ is the internal efficiency of the expander and $h_{1}$ is the specific enthalpy of the state point 1.

For an ORC system with medium and low temperature waste heat utilization, the internal efficiency of the expander $\eta_{t}$ varies little because of the relatively small change of temperature and pressure during the cycle.

In order to facilitate the subsequent thermodynamic calculation and analysis of the thermodynamic performance of the system, it is considered that $\eta_{t}$ has a fixed value.

Based on $h_{2}$ and $p_{2}$, other state parameters of the state point 3 are determined and given by

$$
\begin{aligned}
& T_{3}=\operatorname{refpropm}\left(' T^{\prime}, H^{\prime}, h_{2},{ }^{\prime} P^{\prime}, P_{2}, \text { FLUID }\right) \\
& S_{2}=\operatorname{refpropm}\left({ }^{\prime} S^{\prime},{ }^{\prime} H^{\prime}, h_{2},{ }^{\prime} P^{\prime}, P_{2}, F L U I D\right)
\end{aligned}
$$

The capacity of the expander to do work is expressed by

$$
w_{t}=h_{1}-h_{2}
$$

\subsubsection{Working fluid circulating pump part}

After the condenser condenses, the low-temperature liquid working fluid enters the working fluid circulation pump. The corresponding state point on the T-S diagram is 6 . The state point of the working fluid in the pump after adiabatic pressurization is 8 . The state point of the working fluid after isentropic compression in the ideal state is 7 . For the state point 7 ,

$$
S_{7}=S_{6}, P_{7}=P_{\text {evap }}
$$

where $P_{\text {evap }}$ is the evaporation pressure of the system $(\mathrm{kPa})$ and $P_{8}=P_{\text {evap }}$.

Other state parameters of the state point 7 are given by

$$
\begin{gathered}
T_{7}=\operatorname{refpropm}\left({ }^{\prime} T^{\prime},{ }^{\prime} P^{\prime}, P_{7},{ }^{\prime} S^{\prime}, S_{7}, F L U I D\right) \\
h_{7}=\operatorname{refpropm}\left({ }^{\prime} H^{\prime}, ' T^{\prime}, T_{7}, S^{\prime}, S_{7}, F L U I D\right)
\end{gathered}
$$


Based on the adiabatic compression efficiency $\eta_{p}$ of the working fluid pump, the specific enthalpy of the state point 8 can be obtained as

$$
h_{8}=h_{6}-\left(h_{7}-h_{6}\right) / \eta_{p}
$$

Based on $h_{8}$ and $P_{8}$, other state parameters of the state point 8 are determined and given by

$$
\begin{aligned}
& T_{8}=\operatorname{refpropm}\left(' T^{\prime},{ }^{\prime} H^{\prime}, h_{8},{ }^{\prime} P^{\prime}, P_{8}, F L U I D\right) \\
& S_{8}=\operatorname{refpropm}\left({ }^{\prime} S^{\prime},{ }^{\prime} H^{\prime}, h_{8},{ }^{\prime} P^{\prime}, P_{8}, F L U I D\right)
\end{aligned}
$$

In an ORC system with medium and low temperature waste heat utilization, the consumption power of the refrigerant circulating pump is not small in proportion to the output power of the expander. When calculating the external output power of the ORC system, the consumption of the working fluid circulating pump cannot be neglected, and is given by

$$
w_{p}=h_{8}-h_{6}
$$

\subsubsection{Evaporator part}

The evaporator of the ORC system can be divided into three sections: preheating section, evaporating section and superheating section. Generally, the section of the working fluid preheated to the saturated liquid from the outlet state of the circulating pump is defined as the preheating section. The working fluid in the state point 8 heated after the preheating section reaches the state 9 of the inlet of the evaporation section. After the heat medium releases heat through the preheating section, its state point changes from 13 to 14. For the state point 9 ,

$$
T_{9}=T_{\text {evap }}, Q=0
$$

where $T_{\text {evap }}$ is the evaporation temperature of the system (K) and $Q$ is the dry gas liquid mixture of working fluid $(\mathrm{kg} / \mathrm{kg})$.

Other state parameters of the state point 9 are given by

$$
\begin{aligned}
& h_{9}=\operatorname{refpropm}\left({ }^{\prime} H^{\prime},{ }^{\prime} T^{\prime}, T_{9},{ }^{\prime} Q^{\prime}, 0, F L U I D\right) \\
& S_{9}=\operatorname{refpropm}\left({ }^{\prime} S^{\prime},{ }^{\prime} T^{\prime}, T_{9},{ }^{\prime} Q^{\prime}, 0, F L U I D\right)
\end{aligned}
$$

In the T-S diagram, $T_{P P T D}$ represents the node temperature difference of the evaporator, which is an important design parameter of the evaporator. Usually the minimum value is $3^{\circ} \mathrm{C} \mathrm{C}-7^{\circ} \mathrm{C}$. If the heat source is sufficient, the node temperature difference of the evaporator can be moderately increased to reduce the heat exchanger area and system cost.
Therefore, the temperature of the state point 13 is given by

$$
T_{13}=T_{\text {evap }}+T_{P P T D}
$$

Using medium-low temperature and high pressure air to simulate the heat source, when the air temperature is high, the specific enthalpy is mainly affected by temperature, but hardly by pressure. Therefore, the pressure of heat source can be maintained at atmospheric pressure $P_{a m b}$. The specific enthalpy of the state point 13 is determined according to the temperature of the heat source:

$$
\begin{aligned}
& h_{13}=\operatorname{refpropm}\left({ }^{\prime} H^{\prime}, T^{\prime} T^{\prime}, T_{13},{ }^{\prime} P^{\prime}, P_{a m b}, G A S\right) \\
& S_{13}=\operatorname{refpropm}\left({ }^{\prime} S^{\prime},{ }^{\prime} T^{\prime}, T_{13},{ }^{\prime} H^{\prime}, h_{13}, G A S\right)
\end{aligned}
$$

According to the law of conservation of energy, the heat absorbed by working fluids in the preheating section is

$$
Q_{e 1}=m_{h}\left(h_{13}-h_{14}\right)=m_{f}\left(h_{9}-h_{8}\right)
$$

where $m_{h}$ is the mass flow rate of the heat source $(\mathrm{kg} / \mathrm{s})$ and $m_{f}$ is the mass flow rate of the working fluid $(\mathrm{kg} / \mathrm{s})$.

After the circulating working fluid in the preheating section is saturated, the working fluid continues to absorb heat and change phase in the evaporator. The evaporation section is usually defined by the point where the saturated liquid medium undergoes a phase transition and is heated to saturated vapor.

The saturated liquid working fluid with the state point 9 is heated through the evaporation section to reach the inlet state of the superheated section 10. After the heat medium releases heat through the preheating section, its state point changes from 12 to 13 . For the state point 10 ,

$$
T_{10}=T_{\text {evap }}, Q=1
$$

Other state parameters of the state point 10 are given by

$$
\begin{aligned}
& h_{10}=\operatorname{refpropm}\left({ }^{\prime} H^{\prime},{ }^{\prime} T^{\prime}, T_{10},{ }^{\prime} Q^{\prime}, 1, F L U I D\right) \\
& S_{10}=\operatorname{refpropm}\left({ }^{\prime} S^{\prime}, T^{\prime} T^{\prime}, T_{10},{ }^{\prime} Q^{\prime}, 1, F L U I D\right)
\end{aligned}
$$

According to the law of conservation of energy, the heat absorbed by the working fluid in the evaporation section is given by

$$
Q_{e 2}=m_{h}\left(h_{12}-h_{13}\right)=m_{f}\left(h_{10}-h_{9}\right)
$$

The outlet of the refrigerant in the evaporator section of the evaporator is saturated vapor. After heating through the superheated section [9], the organic working quality becomes overheated. This section is usually defined as the 
overheating section. The saturated steam working state with the state point 10 is heated through the superheated section to reach the inlet state of the expander 1 . After the heat medium releases heat through the superheated section, its state point changes from 13 to 14 . In the T-S diagram, the superheat of the working fluid is $T_{\text {sup }}$. For the state point 1 ,

$$
T_{1}=T_{\text {evap }}+T_{\text {sup }}, P_{1}=P_{\text {evap }}
$$

Other state parameters of the state point 1 are given by

$$
\begin{aligned}
& h_{1}=\operatorname{refpropm}\left({ }^{\prime} H^{\prime}, T^{\prime}, T_{1},{ }^{\prime} P^{\prime}, P_{\text {evap }}, F L U I D\right) \\
& S_{1}=\operatorname{refpropm}\left({ }^{\prime} S^{\prime}, ' T^{\prime}, T_{1},{ }^{\prime} P^{\prime}, P_{\text {evap }}, F L U I D\right)
\end{aligned}
$$

According to the law of conservation of energy, the heat absorbed by the working fluid in the superheated section is given by

$$
Q_{e 3}=m_{h}\left(h_{11}-h_{12}\right)=m_{f}\left(h_{1}-h_{10}\right) .
$$

\subsubsection{Condenser part}

The condenser of ORC system can also be divided into three sections [10]: precooling section, condensing section and supercooling section. The superheated working fluid vapor discharged from the tail of the expander is cooled to saturated vapor at the initial stage of the condenser. This initial stage is usually defined as the precooling section. The working state with the state point 2 is cooled by the precooling section to reach the inlet state of the condensing section 4. After absorption heat of the cooling medium in the precooling section, its state point changes from 17 to 18. For the state point 4 ,

$$
T_{4}=T_{\text {cond }}, Q=1
$$

where $T_{\text {cond }}$ is the condensing temperature (K).

Other state parameters of the state point 4 are given by

$$
\begin{aligned}
& h_{4}=\operatorname{refpropm}\left({ }^{\prime} H^{\prime},{ }^{\prime} T^{\prime}, T_{4},{ }^{\prime} Q^{\prime}, 1, F L U I D\right) \\
& S_{4}=\operatorname{refpropm}\left({ }^{\prime} S^{\prime},{ }^{\prime} T^{\prime}, T_{4},{ }^{\prime} Q^{\prime}, 1, F L U I D\right)
\end{aligned}
$$

In general, the minimum temperature difference between refrigerant and refrigerant in condenser is defined as the node temperature difference of condenser $T_{\text {cond }}$. Smaller nodal temperature difference is beneficial to reduce the irreversible loss of the condensation process, but it also increases the heat transfer area of the condenser [11]. The temperature difference of condenser is generally taken as $3^{\circ} \mathrm{C}-7^{\circ} \mathrm{C}$.

The hydraulic medium is used as the cooling medium of the system, and the temperature at the state point I7 is

$$
T_{17}=T_{\text {cond }}-T_{\text {cond }}
$$

Other state parameters of the state point 17 are given by

$$
\begin{gathered}
h_{4}=\operatorname{refpropm}\left({ }^{\prime} H^{\prime}, ' T^{\prime}, T_{17}, P^{\prime}, P_{\text {water }}, \text { WATER }\right) \\
S_{17}=\operatorname{refpropm}\left({ }^{\prime} S^{\prime},{ }^{\prime} T^{\prime}, T_{17},{ }^{\prime} H^{\prime}, h_{17}, \text { WATER }\right)
\end{gathered}
$$

According to the law of conservation of energy, the heat released by the working fluid in the precooling section is

$$
Q_{c 1}=m_{c}\left(h_{18}-h_{17}\right)=m_{f}\left(h_{2}-h_{4}\right)
$$

where $m_{c}$ is the mass flow rate of cooling water $(\mathrm{kg} / \mathrm{s})$.

The saturated vapor state of organic working fluid in the condenser continues to cool to saturated liquid, which is usually defined as the condensation section. The tail exhaust temperature of the ORC system is low when using medium and low temperature waste heat. The condensation heat of the cooling medium usually occupies most of the condenser load, so the condensation section area is relatively large.

The working fluid with the state point 4 reaches the inlet state 5 of the supercooling section after cooling through the precooling section. After absorption heat of the cooling medium in the precooling section, its state point changes from 16 to 17 . For the state point 5 ,

$$
T_{5}=T_{\text {cond }}, Q=0 .
$$

Other state parameters of the state point 5 are given by

$$
\begin{gathered}
h_{5}=\operatorname{refpropm}\left({ }^{\prime} H^{\prime}, T^{\prime}, T_{5},{ }^{\prime} Q^{\prime}, 0, F L U I D\right) \\
S_{5}=\operatorname{refpropm}\left({ }^{\prime} S^{\prime},{ }^{\prime} T^{\prime}, T_{5},{ }^{\prime} Q^{\prime}, 0, F L U I D\right)
\end{gathered}
$$

According to the law of conservation of energy, the heat released by the working fluid in the condensation section is given by

$$
Q_{c 2}=m_{c}\left(h_{17}-h_{16}\right)=m_{f}\left(h_{4}-h_{5}\right)
$$

In order to ensure the safe operation of the refrigerant pump, the working fluid at the pump inlet can have a smaller degree of undercooling. The supercooling section is usually defined as the section where saturated liquid organic refrigerants continue to be cooled to the supercooling state in the condenser. The proportion of supercooling section to the whole condenser is also very small. 
The working fluid with the state point 5 reaches the state 6 of the circulating pump inlet after cooling through the supercooling section. After absorption heat of the cooling medium in the supercooling section, its state point changes from 15 to 16 . For the state point 6 ,

$$
T_{6}=T_{\text {cond }}-T_{\text {sub }}, P_{6}=P_{\text {cond }}
$$

Other state parameters of the state point 6 are given by

$$
\begin{aligned}
& h_{6}=\operatorname{refpropm}\left({ }^{\prime} H^{\prime},{ }^{\prime} T^{\prime}, T_{6},{ }^{\prime} P^{\prime}, P_{\text {cond }}, F L U I D\right) \\
& S_{6}=\operatorname{refpropm}\left(' S^{\prime},{ }^{\prime} T^{\prime}, T_{6},{ }^{\prime} P^{\prime}, P_{\text {cond }}, F L U I D\right)
\end{aligned}
$$

The heat released by working fluid in the undercooled section is given by

$$
Q_{c 3}=m_{c}\left(h_{16}-h_{15}\right)=m_{f}\left(h_{5}-h_{6}\right)
$$

\subsection{Performance evaluation model of ORC system}

From the point of view of energy analysis and exergy analysis, based on the first law of thermodynamics and the second law of thermodynamics, an evaluation model [12] is established for an ORC system with medium and low temperature waste heat utilization.

\subsubsection{Evaluation index of system performance based on the first law of thermodynamics}

The ORC system is a kind of thermodynamic system for recovering and utilizing medium and low-grade waste heat resources. Its net output function can directly reflect the production capacity of the system, and it is also one of the most important evaluation indexes of the system. Based on the analysis of the above component model, we can see the following: the output specific work of expander is $w_{t}$, and the consumption specific work of working fluid circulating pump is $w_{p}$. Therefore, the actual net output work of the ORC system is

$$
W_{n e t}=m_{f}\left(w_{t}-w_{p}\right)
$$

Thermal efficiency is another important evaluation index of ORC system based on the first law of thermodynamics [13]. It is also known as the efficiency of the first law of thermodynamics. It can reflect the proportion of the energy gain of the system to the total energy consumption. The thermal efficiency of the ORC system is defined as the ratio of net output power of the system to the absorption of heat by the organic matter from the heat source, which is given by

$$
\eta_{t h}=\frac{W_{n e t}}{Q_{e}},
$$

where $Q_{e}$ is the total heat absorbed by the organic working medium from the heat source medium, and

$$
Q_{e}=Q_{e 1}+Q_{e 2}+Q_{e 3}
$$

The heat transfer area per unit net output power of the system is another evaluation index derived from the net output power of the system. The index is used to calculate the working capacity of the system while taking into account the economic factor of the system heat exchanger. In fact, the heat exchanger is not only one of the core pieces of equipment of an ORC system, but also the equipment with the highest electricity cost. Its investment cost accounts for more than $80 \%$ of the initial investment of the ORC system. In order to improve the economy of the ORC system, it is necessary to reduce the heat transfer area required for the net output power of the system.

The heat transfer area of an ORC system heat exchanger is given by

$$
A_{i}=\frac{Q_{i}}{K_{i} \Delta t_{i}}
$$

where $Q_{i}$ is the heat transfer of the heat exchanger, $K_{i}$ is the heat transfer coefficient of the heat exchanger, related to fluid and process on both sides of the heat exchanger, and $\Delta t_{i}$ is the mean temperature difference in heat transfer process. The logarithmic mean temperature difference is usually adopted in actual calculation.

Therefore, the heat transfer area per unit net output of the ORC system is given by

$$
A_{\text {net }}=\frac{A}{W_{\text {net }}}
$$

\subsubsection{Evaluation index of system performance based on second law of thermodynamics}

From the first law of thermodynamics, heat and work are equivalent in quantity. But from the second law of thermodynamics, there is a difference in quality between heat and work. Therefore, it is necessary to establish a supplementary evaluation index according to the second law of thermodynamics. Exergy is such a concept based on the second law of thermodynamics. It is defined as follows. In a reversible process, energy can be transformed into a useful part. An actual process must be irreversible, and it is impossible to obtain the greatest degree of conversion work, 
so in an actual process possible devaluation, i.e. "exergy loss”, will inevitably appear. Exergy loss can directly reflect the power loss of each part of the system caused by irreversible factors. It can also reflect the power loss of the whole ORC system. Similar to energy analysis, exergy analysis can be added to determine the exergy loss of each component.

The exergy loss of the expansion part is given by

$$
I_{t}=m_{f} T_{a m b}\left(s_{2}-s_{1}\right)
$$

The exergy loss of the working fluid circulating pump is given by

$$
I_{p}=m_{f} T_{a m b}\left(s_{8}-s_{6}\right)
$$

The exergy loss of the evaporator part is given by

$$
I_{\text {evap }}=m_{f} T_{a m b}\left[\left(s_{1}-s_{8}\right)-\frac{h_{1}-h_{8}}{T_{h}}\right]
$$

where $T_{h}$ is the mean temperature of the high temperature heat source.

The exergy loss of the condenser part is given by

$$
I_{\text {cond }}=m_{f} T_{a m b}\left[\left(s_{6}-s_{2}\right)-\frac{h_{6}-h_{2}}{T_{t}}\right]
$$

where $T_{t}$ is the mean temperature of the cryogenic coolant

According to the exergy loss distribution of components in the system, the total exergy loss of will inevitably appear he ORC system is obtained by

$$
\begin{aligned}
I_{\text {total }} & =I_{t}+I_{p}+I_{\text {evap }}+I_{\text {cond }} \\
& =m_{f} T_{\text {amb }}\left[\frac{h_{8}-h_{1}}{T_{h}}-\frac{h_{6}-h_{2}}{T_{t}}\right]
\end{aligned}
$$

In addition, according to the second law of thermodynamics, the efficiency of the second law of thermodynamics can be obtained. The efficiency of the second law of thermodynamics is also called the exergy efficiency. It can reflect the degree of effective utilization of waste heat during recycling. The efficiency of the second law of thermodynamics is defined as the ratio of increased revenue to energy consumed, which is expressed by

$$
\eta=\frac{W_{\text {net }}}{Q_{e}\left(1-\frac{T_{l}}{T_{h}}\right)}
$$

In this paper, the basic working principle of the ORC system is first described, and its main components are introduced. The ideal organic Rankine cycle can be realized by four thermodynamic processes: constant pressure heating, adiabatic expansion, constant pressure cooling and adiabatic pressurization. The corresponding system components are evaporator, expander, condenser and working fluid circulating pump. Then, by comparing with the Rankine cycle, the main characteristics of the organic Rankine cycle are analyzed.

Based on the first law of thermodynamics and the second law of thermodynamics, the model of the main components of the system, such as expander, circulating pump, evaporator and condenser, is established in a MATLAB environment. Considering the combination of the energy analysis and exergy analysis of the system, which can reflect the performance of the system more comprehensively, the evaluation model of ORC system for medium and low temperature waste heat utilization is established. The main evaluation indexes of the model include: net output work, thermal efficiency, heat transfer area per unit net output work, system exergy loss, and the efficiency of the second law of thermodynamics.

The sensitivity analysis of the system is carried out based on the first law of thermodynamics and the second law of thermodynamics [15]. The effects of the key operating parameters, such as evaporation temperature, superheating degree, condensation temperature, and supercooling degree, on the performance of ORC system for medium and low temperature waste heat utilization were studied. The sensitivity analysis results show that it is necessary to select the optimum evaporation temperature, minimize the superheating, and reduce the condensation temperature, the combination of which has a great positive effect on improving the performance of the system. Considering the total exergy loss of the system, the total exergy loss will decrease with increased evaporation temperature and superheating. The goal of reducing heat transfer area per unit net output power can be achieved by choosing the optimum evaporation temperature, reducing superheating and supercooling, and decreasing the condensation temperature.

Through the sensitivity analysis of ORC system, it can be seen that the key operating parameters such as evaporation temperature, superheat degree, condensation temperature, and supercooling degree have a certain degree of influence on the performance of the system. Therefore, in order to maximize the thermal efficiency of the ORC system, a search method is needed to optimize the system parameters and obtain the best combination of working parameters. At present, the ant colony algorithm (ACO) has been widely used as a parallel and efficient global random search algorithm in the field of parameter optimization. It draws lessons from the genetic mechanism and natural selection rules in the biological field. In this paper, the ant colony algorithm is used to optimize the parameters of ORC system. 


\subsection{Solving multi-objective parameters of ORC cycle based on improved ant colony algorithm}

The Ant Colony Algorithm (ACA) is a bionic algorithm inspired by M. Dorigo et al. in 1991 by analyzing an ant colony's foraging process in nature, i.e. the cooperative behavior among simple ants evolves into the high social behavior of the colony. The idea is to find the optimal path between ants by the positive feedback mechanism of pheromone on the route.

A multi-objective optimization problem is a kind of universal problem in scientific research and engineering practice. Its object of study is the optimization problem of more than one objective function in a given region. It can be described as

$$
\min f(x)=\left(f_{1}(x), f_{2}(x), \cdots, f_{n}(x)\right) \text { s.t } x \in S
$$

where $f_{i}(x)(i=1,2, \cdots, n)$ is the objective function for $\mathrm{n}$ and $S$ is the D-dimensional feasible space. For the multiobjective optimization problem, there is no good or bad distinction between each sub-goal, no better solution than at least one of them and no other objective is bad, and the elements in the solution set are not comparable to each other for all objectives.

However, when the ant colony algorithm is used to solve multi-objective optimization problems, the positive feedback pheromone communication between ants will make the ant colony tend to concentrate in a certain area of feasible space, so it is not conducive to the solution of multi-objective problems. In this paper, an ant colony algorithm for solving multi-objective optimization problems is proposed based on the characteristics of such problems.

Assume the number of cities is $N$, the number of ants is $M$. At time $t$ the transfer probability $P_{i j}^{k}(t)$ of the $k$ th ant moving from city $i$ to city $j$ is expressed by

$$
P_{i j}^{k}(t)=\left\{\begin{array}{l}
\frac{\tau_{i j}^{\alpha} \cdot \eta_{i j}^{\beta}(t)}{\sum_{\text {leallowed }} \tau_{t}^{\alpha} \cdot \eta_{i j}^{\beta}(t)} j \in \text { allowed } \\
0 \quad \text { else }
\end{array}\right.
$$

where $k=1,2, \cdots, M, i, j=1,2, \cdots, N$ is the pheromone on the path between city $i$ and city $j, \eta_{i j}(t)$ is the heuristic information, $\eta_{i j}(t)=1 / d_{i j}\left(d_{i j}\right.$ is the distance between cities $i j$ ), $\alpha$ and $\beta$ are heuristic factors, and allowed is the set of cities that ant $k$ has never gone through until time $t$.

Ants leave a pheromone on every path. In order to avoid local optimization, a pheromone volatilization mechanism is introduced. Update of the pheromone is carried out in the following way:

$$
\tau_{i j}(t+1)=\rho \cdot \eta_{i j}(t)+\Delta \tau_{i j}(t),
$$

$$
\Delta \tau_{i j}(t)=\sum_{k=1}^{M} \Delta \tau_{i j}(t),
$$

where $\rho$ is the volatilization coefficient and $\rho \in[0,1]$, $\Delta \tau_{i j}(t)$ is the pheromone left by the $k$ th ant on the path and is a function of the total path length.

For single-objective optimization problems, the search mechanism based on transfer probability and pheromone updating method of basic ant colony algorithm is helpful to guide the ant colony to search for the area with high pheromone concentration so that ants can gather in a certain area of the space and eventually find the global optimal solution. However, for multi-objective optimization problems, the solution is relative and there is no absolute optimum. Therefore, the Pareto solution set is usually obtained. Since all the points in the Pareto solution set can be regarded as the optimal solution of the designer, it is required that the obtained non-inferior solution set should be as close as possible to the real Pareto solution set of the problem. The obtained Pareto front should have better uniformity and dispersion range.

Based on the above analysis, a new definition for the pheromone update method of the basic ant colony algorithm is proposed $[14,15]$. Because the pheromone is in a continuous domain, it is different from the case in a discrete domain, so that the pheromone is left only at the ant's position. In order to guide ants to search for feasible space better, an external set $A(t)$ is used to store the Pareto solution set found until time $t$. If an ant enters the set $A(t)$, this indicates that the location of the ant is incontrollable. The pheromones of ants should be added so that other ants can search the neighborhood of the ant's location. On the other hand, if the fails to enter the set $A(t)$, the pheromone level should be reduced appropriately. When multiple ants enter the set $A(t)$ at the same time, the pheromone increment of each ant should be distinguished. For the uniformity of the Pareto frontier, the minimum distance between the new ant and the objective function of the solution in the original set $A(t)$ is taken as the pheromone released by the ant in its position. The greater the distance, the higher the pheromone content, and the higher the probability of other ants to search for the ant's location neighborhood. Therefore, the pheromone update is

$$
\tau_{i}(t+1)= \begin{cases}\rho \cdot \tau_{i}(t)+\delta(t) & \text { if }(x \in A(t+1)) \\ \rho \cdot \tau_{i}(t) & \text { else }\end{cases}
$$

where $\delta(t)=\min \sqrt{\sum_{i=1}^{n}\left(f_{i}(x)-f_{i}(v)\right)}, x_{v} \in A(t)$, and $\rho$ is the volatility coefficient.

The movement of ant $i$ is related to the pheromone and distance of ant $j$. Ants with high pheromone concen- 
tration and distance from each other should be selected as the next move direction with higher probability. Then the transition probability is given by

$$
P_{i j}(t)=\frac{\tau_{j}^{\alpha}(t) \cdot \eta_{i j}^{\beta}(t)}{\sum_{l \in M} \tau_{l}^{\alpha}(t) \cdot \eta_{i l}^{\beta}(t)}
$$

where $\eta_{i j}(t)=1 / d_{i j}$, is the distance between ant $i$ and ant $j$.

Through the above strategy, the ant not only searches the sparse area in the set $A(t)$, but also searches the location of other ants. In order to speed up convergence, a random number-based search mechanism is also introduced. For a given constant in $[0,1]$, when the random number is $z(\in[0,1]) \leq w$, it moves according to the above transfer probability. When $z>w$, the solution selected arbitrarily from the set $A(t)$ is taken as the next move direction for ant $i$, and the neighborhood of the solution is searched.

The ant colony algorithm for solving multi-objective optimization problem is as follows.

1. Initialize the location and the set $A(t)$ of ants in search space $S$ and let $t=0$.

2. $t=t+1$.

3. Generate random number $z$. If $z \leq w$, search according to Eq. (61). If $z>w$, the arbitrarily selected solution in the set is used as the next move direction to do the neighborhood search.

4. Update the set $A(t)$.

5. Update pheromone content of ants according to Eq (60).

6. If is greater than the maximum number of iterations, exit; otherwise, go to step (2).

When using the ant colony algorithm to solve multiobjective optimization problems, it is hoped that the population of the algorithm can maintain the diversity of the population in the iterative process, which is different from the single objective optimization when clustering near the optimal point. In this section, a preliminary analysis of the population diversity of the algorithm will be given.

Let be the maximum diffusion range of the real Pareto solution set for multi-objective optimization problem and assume ants can enter the solution set $A(t)$ every time at the A time, then

$$
\lim _{t \rightarrow \infty} \tau_{i}(t)=\tau \leq \frac{\delta_{\max }}{1 \rho} .
$$

The decomposition based multi-objective ant colony optimization is to divide the ant colony into multiple subgroups and assign them to various targets. Each subgroup maintains a pheromone matrix, and each ant has its own heuristic information matrix [16-22]. Paretobased ant colony optimization (PACO) uses multiple pheromone matrices and a single heuristic information matrix. Population-based multi-objective ant colony optimization uses multiple pheromone matrices and multiple heuristic information matrices.

Although the multi-objective ant colony optimization algorithm has been used to solve many multi-objective optimization problems, there are still some defects. There are many studies on the convergence analysis of singleobjective ant colony optimization algorithm, but few on the convergence analysis of multi-objective, especially on the convergence analysis of Pareto solution domain. The experimental analysis of the multi-objective ant colony algorithm is usually carried out on a two-dimensional multiobjective, but the research on complex multi-objective optimization problems is very sparse. Therefore, these are the areas where multi-objective ant colony optimization algorithm needs to be improved, and further research is needed.

\section{Results}

In order to verify the effectiveness of the proposed multiobjective performance optimization method for ORC cycle based on improved ant colony algorithm, the experiment is carried out. The experimental environment is a machine with 48GB memory running the Windows 7 operating system. The multi-objective performance optimization method of ORC cycle based on the improved ant colony algorithm is compared with the multi-objective optimization method of the traction power supply system based on improved particle swarm optimization and the thermal performance optimization method based on low-temperature geothermal cascade split-flow organic Rankine cycle system. The experimental results are shown in Table 1. A represents the proposed method, B represents the multiobjective optimization method for the traction power supply system based on improved particle swarm optimization, and $\mathrm{C}$ represents the thermal performance optimization method for low-temperature geothermal cascade splitflow organic Rankine cycle system.

From Table 1 it can be seen that the efficiency of performance optimizations of the 3 methods also change with increasing number of objectives. When the number of objectives is 20 , the performance optimization efficiency of the proposed method is $100 \%$, the performance optimization efficiency based on improved particle swarm optimization is $98.25 \%$, and the performance optimization efficiency 
Table 1: Comparison experiments of different methods for performance optimization efficiency

\begin{tabular}{cccc}
\hline $\begin{array}{c}\text { Number of } \\
\text { objectives }\end{array}$ & \multicolumn{3}{c}{ Performance optimization efficiency /\% } \\
\hline 20 & A & B & C \\
\hline 40 & 990.56 & 98.25 & 96.89 \\
60 & 98.78 & 97.89 & 96.12 \\
80 & 98.32 & 96.45 & 95.35 \\
100 & 97.99 & 95.12 & 94.68 \\
120 & 97.56 & 94.23 & 92.68 \\
\hline
\end{tabular}

based on low-temperature geothermal cascade shunt is $96.89 \%$. The comparison shows that the performance optimization efficiency of the proposed method is $1.75 \%$ and $3.11 \%$ higher than that of the improved particle swarm optimization method and the low-temperature geothermal cascade shunt optimization method, respectively.

When the number of objectives is 40 , the performance optimization efficiency of the proposed method is $99.56 \%$, the performance optimization efficiency based on improved particle swarm optimization is $97.89 \%$, and the performance optimization efficiency based on lowtemperature geothermal cascade shunt is $96.12 \%$. The comparison shows that the performance optimization efficiency of the proposed method is $1.67 \%$ and $3.44 \%$ higher than that of the improved particle swarm optimization method and the low-temperature geothermal cascade shunt optimization method, respectively.

When the number of objectives is 60 , the performance optimization efficiency of the proposed method is $98.78 \%$, the performance optimization efficiency based on improved particle swarm optimization is $97.21 \%$, and the performance optimization efficiency based on lowtemperature geothermal cascade shunt is $95.35 \%$. The comparison shows that the performance optimization efficiency of the proposed method is $1.57 \%$ and $3.43 \%$ higher than that of the improved particle swarm optimization method and the low-temperature geothermal cascade shunt optimization method, respectively.

When the number of objectives is 80 , the performance optimization efficiency of the proposed method is $98.32 \%$, the performance optimization efficiency based on improved particle swarm optimization is $96.45 \%$, and the performance optimization efficiency based on lowtemperature geothermal cascade shunt is $94.68 \%$. The comparison shows that the performance optimization efficiency of the proposed method is $1.87 \%$ and $4.64 \%$ higher than that of the improved particle swarm optimiza- tion method and the low-temperature geothermal cascade shunt optimization method, respectively.

When the number of objectives is 100, the performance optimization efficiency of the proposed method is $97.99 \%$, the performance optimization efficiency based on improved particle swarm optimization is $95.12 \%$, and the performance optimization efficiency based on lowtemperature geothermal cascade shunt is $93.68 \%$. The comparison shows that the performance optimization efficiency of the proposed method is $2.87 \%$ and $4.31 \%$ higher than that of the improved particle swarm optimization method and the low-temperature geothermal cascade shunt optimization method, respectively.

When the number of objectives is 120 , the performance optimization efficiency of the proposed method is $97.56 \%$, the performance optimization efficiency based on improved particle swarm optimization is $94.23 \%$, and the performance optimization efficiency based on lowtemperature geothermal cascade shunt is $92.31 \%$. The comparison shows that the performance optimization efficiency of the proposed method is 3.33\% and 5.25\% higher than that of the improved particle swarm optimization method and the low-temperature geothermal cascade shunt optimization method, respectively.

Experimental results show that the performance optimization method based on low-temperature geothermal cascade shunt has the lowest efficiency, followed by the multi-objective optimization method based on improved particle swarm optimization for traction power supply system. The proposed method has the highest performance optimization efficiency and certain application performance.

The results of comparing the optimization time of the three methods are shown in Figure 1, Figure 2, and Figure 3.

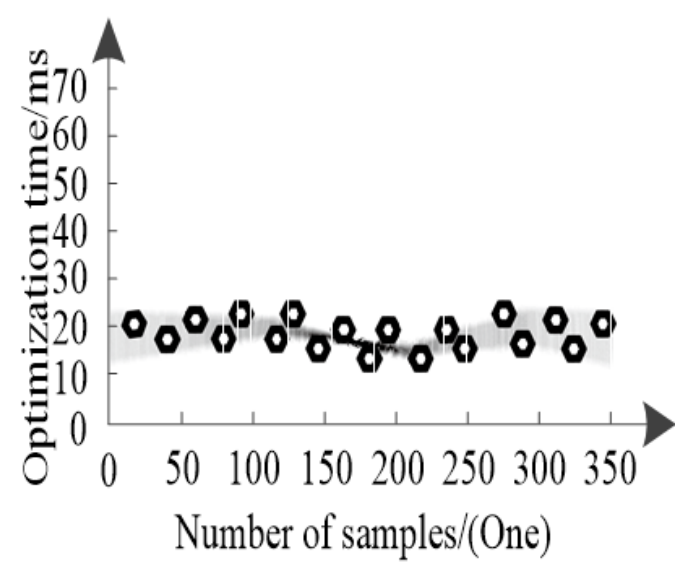

Figure 1: Optimization time of the proposed method 


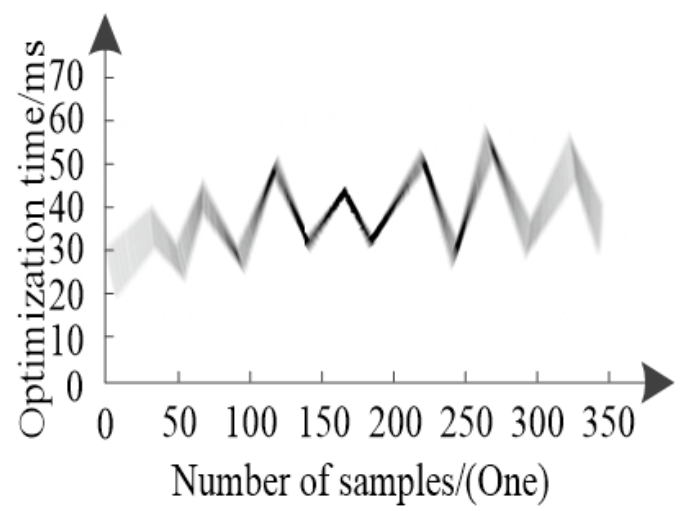

Figure 2: Optimization time of multi-objective optimization method of traction power supply system based on improved particle swarm optimization algorithm

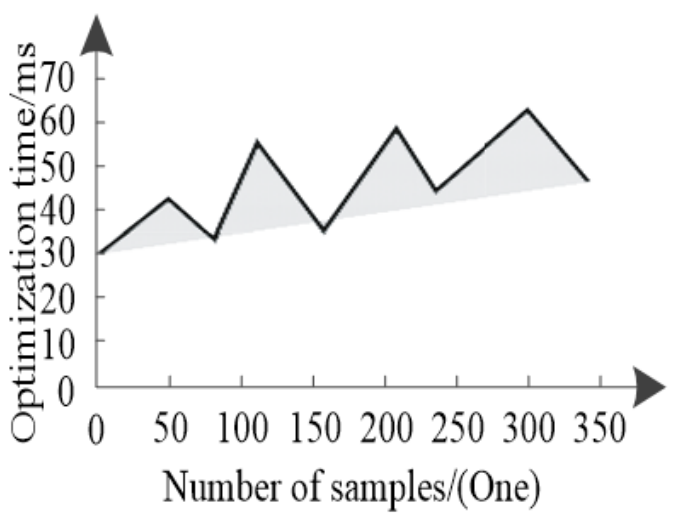

Figure 3: Optimization time of thermal performance optimization of organic Rankine cycle system based on low temperature geothermal cascade diversion

From Figures 1 - 3, it can be seen that, the optimization time of the proposed method is in a relatively stable. The optimization time of the method based on the improved particle swarm optimization algorithm and the low-temperature geothermal cascade method is very unstable. When the number of samples is 50 , the optimization time of the proposed method is $15 \mathrm{~ms}$. The optimization time of the improved particle swarm-based optimization algorithm is $22 \mathrm{~ms}$, and the optimization time of the low-temperature geothermal cascade flow optimization algorithm is $32 \mathrm{~ms}$. The proposed method is $7 \mathrm{~ms}$ and $17 \mathrm{~ms}$ lower than the other two methods respectively.

When the number of samples is 350 , the optimization time of the proposed method is $11 \mathrm{~ms}$. The optimization time of the improved particle swarm-based optimization algorithm is $27 \mathrm{~ms}$, and the optimization time of the lowtemperature geothermal cascade flow optimization algorithm is $45 \mathrm{~ms}$. The proposed method is $16 \mathrm{~ms}$ and $34 \mathrm{~ms}$ lower than the other two methods respectively. Through the two sets of comparison data, it can be seen that the optimization time of the proposed method is significantly lower than the other two methods. Therefore, the proposed method effectively reduces the optimization time.

For the proposed multi-objective performance optimization method for the ORC cycle based on the improved ant colony algorithm, a comparison experiment is carried out on the operation efficiency before and after optimization. The experimental results are shown in Table 2. In Table 2, Be represents the model before optimization, and Af represents the model after optimization.

Table 2: Comparison of operation efficiency before and after optimization

\begin{tabular}{ccc}
\hline $\begin{array}{c}\text { Number of } \\
\text { objectives }\end{array}$ & \multicolumn{2}{c}{ Model operation efficiency /\% } \\
Be & Af \\
\hline 20 & 88.92 & 99.82 \\
40 & 86.47 & 98.91 \\
60 & 84.35 & 98.63 \\
80 & 83.12 & 98.20 \\
100 & 82.01 & 97.89 \\
120 & 80.25 & 97.47 \\
\hline
\end{tabular}

From Table 2, it can be seen that, the operation efficiency of the model before and after optimization decreases with the increase of the number of objectives. When the number of objectives is 20 , the operation efficiency of the model before optimization is $88.92 \%$, and that of the model after optimization is $99.82 \%$. The operation efficiency increased by $10.9 \%$ compared with the model before optimization. When the number of objectives is 80 , the operation efficiency of the model before optimization is $83.12 \%$, and that of the model after optimization is $98.20 \%$. The operation efficiency increased by $15.08 \%$ compared with the model before optimization. It shows that the operation efficiency of the optimized model is obviously improved.

When the number of objectives increases from 40 to 100 , the operation efficiency of the model before optimization changes from $86.47 \%$ to $82.01 \%$, and is reduced by $4.46 \%$. The operation efficiency of the model after optimization changes from $98.91 \%$ to $97.89 \%$, which is reduced by $1.02 \%$. It is shown that not only the operation efficiency is improved, but also the operation is more stable after the optimization of the model. 


\section{Discussion}

From the analysis of Table 1, it can be seen that the 3 methods also change the efficiency of performance optimization with the increase of the number of objectives. The thermodynamic performance optimization method based on low-temperature geothermal cascade shunt for organic Rankine cycle system has the lowest efficiency, followed by the multi-objective optimization method based on improved particle swarm optimization for a traction power supply system. The proposed method has the highest performance optimization efficiency and certain application performance.

From the analysis of Figures 1, 2 and 3, it can be seen that, the optimization time of the proposed method has been in a relatively stable state and that of the other methods is very unstable. The optimization time of the proposed method is obviously lower than the other two methods. The proposed method effectively reduces the optimization time.

From the analysis of Table 2, it can be seen that the operation efficiency of the model before and after optimization decreases with the increase of the number of objectives. When the number of objectives is 20 , the operation efficiency of the model before optimization is $88.92 \%$, and that of the model after optimization is $99.82 \%$. The operation efficiency increased by $10.9 \%$ compared with the model before optimization. When the number of objectives is 80 , the operation efficiency of the model before optimization is $83.12 \%$, and that of the model after optimization is $98.20 \%$. The operation efficiency increased by $15.08 \%$ compared with the model before optimization. It shows that the operation efficiency of the model after optimization is obviously improved.

When the number of objectives increases from 40 to 100 , the operation efficiency of the model before optimization changes from $86.47 \%$ to $82.01 \%$, and is reduced by $4.46 \%$. The operation efficiency of the model after optimization changes from $98.91 \%$ to $97.89 \%$, which is reduced by $1.02 \%$. It is shown that after the optimization of the model, not only the operation efficiency is improved, but also the operation is more stable.

\section{Conclusions}

Aiming at the problem of solving multi-objective performance optimization with improved ant colony algorithm, an ORC cycle multi-objective performance optimization method based on improved ant colony algorithm is proposed in this paper.

The thermodynamic model of an ORC system is built by analyzing the ORC cycle system. According to the first law of thermodynamics and the second law of thermodynamics, an ORC system evaluation model is established in a MATLAB environment.

A sensitivity analysis of the system is carried out by using the system performance evaluation index to obtain the best combination of working parameters. The improved ant colony algorithm is used to optimize the multiobjective performance of the ORC system to obtain the optimal solution.

The experimental results show that the proposed multi-objective performance optimization method based on the ant colony algorithm for ORC cycle has a shorter optimization time and a higher optimization efficiency [6, 14, 15].

Acknowledgement: The scientific and technological planning projects of Henan Province, China (NO. 162102310504)

Key project of science and technology research of Henan Provincial Education Department, China (NO. 15A480002).

\section{References}

[1] Yu S., Zhang S., Agbemabiese L., Zhang F., Multi-stage goal programming models for production optimization in the middle and later periods of oilfield development, Ann. Oper. Res., 2017, 255(1-2), 421-437.

[2] Zhao J.H., Liu Z., Daoa M.T., Reliability optimization using multiobjective ant colony system approaches, Reliab. Eng. Syst. Safe, 2017, 92(1), 109-120.

[3] Cheng R., Jin Y., Olhofer M., Sendhoff B., A reference vector guided evolutionary algorithm for many-objective optimization, IEEE T Evolut. Comput., 2016, 20(5), 773-791.

[4] Li K., Kwong S., Zhang Q., Deb K., Interrelationship-based selection for decomposition multiobjective optimization, IEEE Trans. Cybernetics, 2017, 45(10), 2076-2088.

[5] Chen M.W., Xu C.Y., Huang W.X., Multi-objective optimization design of traction power supply system based on improved particle swarm optimization algorithm, China Rail Sci., 2016, 37(1):85-92.

[6] Li W.K., Optimization of thermal performance of low temperature geothermal cascade split ORC system, J. Eng. Therm. Energ. Power, 2016, 31(10):15-19.

[7] Gutjahr W.J., Pichler A., Stochastic multi-objective optimization: a survey on non-scalarizing methods, Ann. Oper. Res., 2016, 236(2), 475-499.

[8] Akhtar T., Shoemaker C.A., Multi objective optimization of computationally expensive multi-modal functions with rbf surrogates and multi-rule selection, J. Global. Optim., 2016, 64(1), 17-32. 
[9] Li K., Deb K., Zhang Q., Zhang Q., Efficient nondomination level update method for steady-state evolutionary multiobjective optimization, IEEE Trans. Cybernetics, 2017, 47(9), 2838-2849.

[10] Sun Z., Zhang Y., Nie Y., Wei W., Lloret J., Song H., Casmoc: A novel complex alliance strategy with multi-objective optimization of coverage in wireless sensor networks, Wirel. Netw., 2017, 23(4), 1201-1222.

[11] Feliot P., Bect J., Vazquez E., A bayesian approach to constrained single- and multi-objective optimization, J. Global. Optim., 2016, 67(1-2), 1-37.

[12] Trummer I., Koch C., Multi-objective parametric query optimization, Vldb. J., 2016, 45(1), 1-18.

[13] Bortolini M., Faccio M., Ferrari E., Gamberi M., Pilati F., Fresh food sustainable distribution: cost, delivery time and carbon footprint three-objective optimization, J. Food Eng., 2016, 174(85), 56-67.

[14] Cai X., Yang Z., Fan Z., Zhang Q., Decomposition-based-sorting and angle-based-selection for evolutionary multiobjective and many-objective optimization, IEEE Trans. Cybernetics, 2016, 47(9), 2824-2837.

[15] Wang X., Tang L., An adaptive multi-population differential evolution algorithm for continuous multi-objective optimization, Inform. Sci., 2016, 348(2), 124-141.

[16] Nie J.J., Logistics distribution based on ant colony algorithm, the optimal path, Automat. Instrum., 2016, 12(5), 3-5.
[17] Yu S.B., Wu L.D., Zhang X.T. Li C., Ma H.J., Overview of multi feature visualization methods for spatial information networks, J. China Acad. Electron. Inf. Tech., 2018, 14(2), 89-145.

[18] Costamagna A., Drigo M., Martini M., Sona B., Venturino E., A model for the operations to render epidemic-free a hog farm infected by the aujeszky disease, Appl. Math. Nonlin. Sci., 2016, 1(1), 207-228.

[19] Gupta D., Singh G., Thakur N., Bhatia R.S., Evaluation of some novel insecticides, biopesticides and their combinations against peach leaf curl aphid, brachycaudus helichrysi infesting nectarine, J. Environ. Biol., 2017, 38(6), 1275-1280.

[20] Best T., Finney K.N., Ingham D.B., Pourkashanian M., CO2enhanced and humidified operation of a micro-gas turbine for carbon capture, J. Clean. Prod., 2018, 176, 370-381.

[21] Gulmezoglu N., Aytac Z., Kutlu I., Kulan E.G., Gozukara G., Mapping boron and beneficial heavy metal ions for wheat-cultivating soils in turkey's boron-mining zone, Appl. Ecol. Env. Res., 2017, 15(3), 1119-1130.

[22] Lakshminarayana G., Vajravelu K., Sucharitha G., Sreenadh S., Peristaltic slip flow of a bingham fluid in an inclined porous conduit with joule heating, Appl. Math. Nonlin. Sci., 2018, 3(1), 41-54. 